
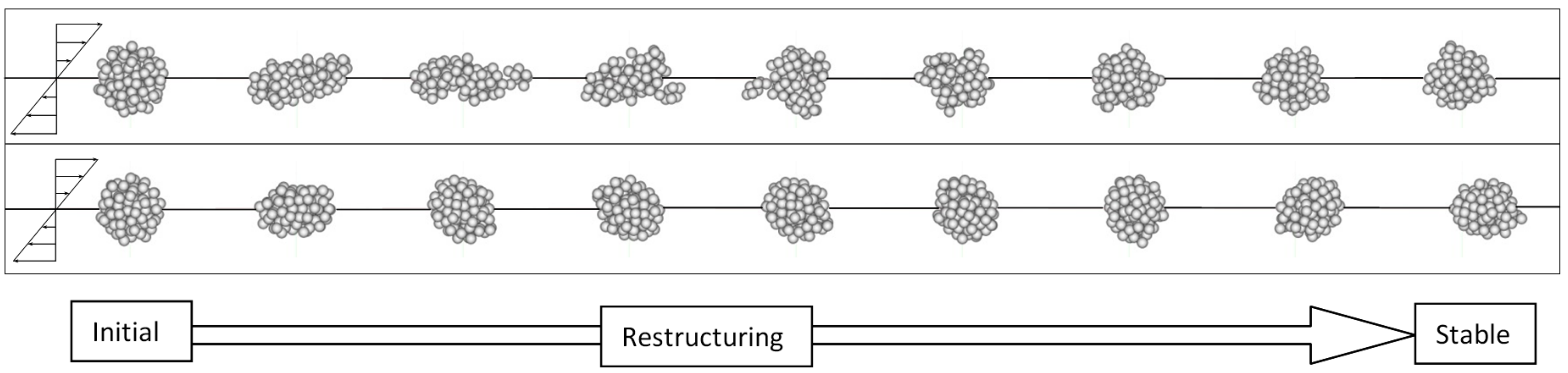


\title{
1 Stability of restructured non-fractal aggregates in simple shear flow
}

\author{
Uyen Tu Lieu*, Shusaku Harada
}

Division of Sustainable Resources Engineering, Faculty of Engineering, Hokkaido University, N13W8, Sapporo 060-8628, Japan

* The email of corresponding author: uyen@trans-er.eng.hokudai.ac.jp Tel.: +81-11-706-6311; Fax: +81-11-706-6311

\section{Abstract}

The restructuring behavior of non-fractal aggregates in simple shear flow is numerically investigated. The change in internal structure of aggregates having different packing properties is examined by Lagrangian simulation method. The many-body hydrodynamic interaction is rigorously estimated by Stokesian dynamics approach while the adhesion of aggregate is manifested via particle-particle interaction. The simulation results show that the restructuring of aggregate originates from the superimposition of rotational and extensional component of simple shear flow. The aggregate rearranges its particles so that a stable structure corresponding to the applied shear flow is obtained. The stable structure is considered as dynamic equilibrium resulting from the balance of the forming and the disintegrating of the bonds between particles. The stable structure of aggregate is dependent strongly on shear condition but weakly on initial structure of aggregate. Despite of the significant difference in the initial packing properties, the stable structure reveals only slightly different. The dependence of stable structure on high shear tress condition is similar for all aggregates. The difference in stable structure of aggregate at low shear stress arises from the irreversible behavior of particle from quasi-stable structure to static structure.

Keywords: aggregate; restructuring; Stokesian dynamics; coordination number 


\section{Introduction}

The aggregation and dispersion processes of colloidal particles are widely seen in various engineering fields such as environmental engineering, material engineering, to name a few. Understanding the behavior of colloidal aggregates in fluid flow is one of the fundamental issues for the prediction and control of the dispersion state of suspension. Population balance model has been developed for quantitative prediction of the size distribution of aggregates from the parent aggregates. In this model, the important parameters on the aggregation and breakup of aggregate are required [1]. Simulation and experimental studies have found that the behaviors of aggregate in flow field, including rigid body, restructuring and breakup, are dependent on the hydrodynamics stress and the strength of aggregate [2-5]. In addition, it is well-known that the aggregate has a wide variety of structures, and the hydrodynamic properties of the aggregates greatly depend on these structures. When the restructuring occurs, the aggregate behaves complexly because the particle-fluid interaction is complicated while the dynamic system has to be taken into account.

Many researchers examined the behavior of aggregate relying on the change in the spacefilling properties i.e. fractal dimension. They found that in shear flow, the aggregates tended to have high fractal dimension by means of experiment [1], numerical simulation [6] and analytical model [7]. Further studies showed that the breakup behavior of the high fractal dimension aggregate was significantly diverse [4,7-9]. One of the reason is that the aggregate with high space-filling properties may have the connectivity of the particle vary, leading to different ways in force distribution and propagation. Moreover, Harada et al. [4] found out that the breakup behavior of non-fractal aggregate (i.e. fractal dimension equals 3) was much more complicated because the aggregate continuously restructured before the breakup. The importance of restructuring has also been reported via the kinetic models of aggregation and 
1 dispersion [10,11]. Although the restructuring of aggregate is critical to the breakup process,

2 little understanding on the restructuring is revealed, even in the case of isolated aggregate.

3 There have only been a few studies on the restructuring dynamics of aggregate in shear

4 flow. Experimental studies such as Blaser [3] confirmed the change in aggregate's structure

5 when the shear was applied. Numerical researches reported that the fractal aggregate

6 restructured and became more dense in terms of volume fraction [12] and radius of gyration

7 [5] for low shear rate. Though the two aforementioned parameters are commonly used to

8 evaluate the compactness and the size of the aggregate, they are quite sensitive to the

9 shape of aggregate [12] and somewhat inappropriate to describe the internal structure.

10 Additionally, the connectivity of non-fractal aggregate becomes more complex during

11 restructuring [4]. Harada et al. [13] showed that the restructuring of dense particle cluster

12 included three stages: firstly the aggregate rearranged to a structure corresponding to shear

13 stress, then the cracks grew inside the aggregate for a long time, finally aggregate irregularly

14 changed toward rupture.

15 The aim of this study is to understand the restructuring behavior of non-fractal aggregates before breakup in simple shear flow by Lagrangian simulation. Since our interest focus on the

17 effect of surrounding flow on the particles, we employ Stokesian dynamics for the calculation 18 of hydrodynamic interaction. The restructuring process of aggregate with different 19 connectivity is performed under various shear flow conditions. We evaluate the restructuring 20 by examining the change in internal structure of the aggregate dynamically. Detail of the 21 effect of the connectivity and flow conditions on the restructuring of aggregate is discussed.

\section{Simulation method}

The full description of the simulation method can be found in the previous studies $[4,13]$. 
1 interaction of multiple primary particles composing the aggregate. The motion of the rigid

2 and spherical particles is simulated by Stokesian dynamics approach [14]. The relation

3 between force, torque, stresslet exerting on particles, and the motion of particles relative to

4 the imposed flow is given in Eq.(1).

$5 \quad\left(\begin{array}{c}\boldsymbol{F} \\ \boldsymbol{T} \\ \boldsymbol{S}\end{array}\right)=\overline{\boldsymbol{R}} \cdot\left(\begin{array}{c}\boldsymbol{U}-\boldsymbol{u}^{\infty} \\ \boldsymbol{\Omega}-\boldsymbol{\Omega}^{\infty} \\ -\boldsymbol{E}^{\infty}\end{array}\right)$

6 where $\boldsymbol{F}, \boldsymbol{T}, \boldsymbol{S}$ are the external force, torque and stresslet acting on particles; $\boldsymbol{U}, \boldsymbol{\Omega}$ the

7 particle translational velocity and rotational velocity; $\boldsymbol{u}^{\infty}, \boldsymbol{\Omega}^{\infty}, \boldsymbol{E}^{\infty}$ the flow velocity, rotational

8 velocity and rate of strain tensor, $\overline{\boldsymbol{R}}$ the grand resistance matrix which is dependent only on

9 the position of the particles. Readers can refer to articles [15-17] for details on the method

10 and calculation of the grand resistance matrix. From Eq.(1), the translational and rotational

11 velocity of particles with prescribed forces and torques are given as follows:

12

$$
\left(\begin{array}{l}
\boldsymbol{U} \\
\boldsymbol{\Omega}
\end{array}\right)=\left(\begin{array}{l}
\boldsymbol{U}^{\infty} \\
\boldsymbol{\Omega}^{\infty}
\end{array}\right)+\left(\begin{array}{ll}
\overline{\boldsymbol{R}}_{F U} & \overline{\boldsymbol{R}}_{F \Omega} \\
\overline{\boldsymbol{R}}_{T U} & \overline{\boldsymbol{R}}_{T \Omega}
\end{array}\right)^{-1} \cdot\left[\left(\begin{array}{c}
\boldsymbol{F} \\
\boldsymbol{T}
\end{array}\right)+\left(\begin{array}{l}
\overline{\boldsymbol{R}}_{F E} \\
\overline{\boldsymbol{R}}_{T E}
\end{array}\right): \boldsymbol{E}^{\infty}\right]
$$

where $\overline{\boldsymbol{R}}_{F U}, \overline{\boldsymbol{R}}_{F \Omega}$, etc. are the components of the grand resistance matrix $\overline{\boldsymbol{R}}$. The instantaneous position of all particles is calculated by updating the matrices in Eq.(2) with each time step. Regarding the simple shear flow characterized by shear rate $\dot{\gamma}$, the surrounding flow field $\boldsymbol{u}^{\infty}, \boldsymbol{\Omega}^{\infty}$ and $\boldsymbol{E}^{\infty}$ are described respectively below.

$$
\boldsymbol{u}^{\infty}(\boldsymbol{r})=\boldsymbol{\Omega}^{\infty} \times \boldsymbol{r}+\boldsymbol{E}^{\infty} \cdot \boldsymbol{r}
$$

$$
\boldsymbol{\Omega}^{\infty}=-\frac{\dot{\gamma}}{2}\left(\begin{array}{l}
0 \\
0 \\
1
\end{array}\right), \quad \boldsymbol{E}^{\infty}=\frac{\dot{\gamma}}{2}\left(\begin{array}{lll}
0 & 1 & 0 \\
1 & 0 & 0 \\
0 & 0 & 0
\end{array}\right)
$$

Figure 1 shows the coordinate system of our calculation and the initial configuration of some non-fractal aggregates. The aggregate is composed of one hundred primary particles. 
1 The particles are assumed smooth and spherical with a diameter of $2 a=650 \mathrm{~nm}$. The

2 properties of particles and fluid are those of polystyrene and ethanol. The particle density, the

3 fluid density and the fluid viscosity are $\rho_{\mathrm{p}}=1056 \mathrm{~kg} / \mathrm{m}^{3}, \rho_{\mathrm{f}}=790 \mathrm{~kg} / \mathrm{m}^{3}$ and $\mu=1.2 \times 10^{-3}$

4 Pa.s, respectively. The particle-particle interaction is calculated from the attractive London-

5 van der Waals potential [18,19]. As the particles approach very close at a few Angstrom,

6 the attractive force is no longer dominant because the other complex phenomena at molecular scale govern the interaction [20]. For this reason, we simply set the attractive force to be zero when the distance of particle's surface is less than $1 \mathbf{n m}$. The overlapping of particles is prevented due to the lubrication effect included in the grand resistance matrix $[14,15]$. Because of the discretization of the simulation, we choose appropriately small time step for each simulation condition to assure that the particles definitely do not overlap. It should be notice that there are some works on the restructuring and breakup of aggregate considering more complex particle interactions such as tangential force when the particles are in contact $[1,6,12,21]$. Though the particle-particle interaction used in our method is simple, we verify that some parameters, which express the behaviors of the aggregate, are relatively in agreement with either experiment study [3] or simulation study employing more complex contact force $[6,21]$. Therefore, the contact force is neglected for the sake of simplicity. In this study, the properties of particle and fluid is appropriately chosen so that the effect of fluid inertia, particle inertia and Brownian motion of particle is neglected due to small Reynolds number, Stokes number and large Péclet number. The detailed discussion on the assumptions, calculation conditions and verification of the simulation are described in the articles $[4,13]$.

The aggregate shown in Fig. 1 is created by employing particle-cluster aggregation model to randomly distributed particles to which an assumed centripetal force is applied. By this method, the isotropy of the particles is obtained in the case that the aggregate is large enough 
1 to neglect the effect of surface particles [22]. The non-fractal property of the aggregate is

2 exhibited via its spherical morphology as can be seen in the figure. The connectivity of

3 aggregate is expressed by coordination number which has been widely investigated as an

4 important packing property of granular media at a local scale [23]. Coordination

5 number is defined as the average number of particle adjacent to a particular one within

designated separation of particle's surface. The chosen separation has to satisfy to

7 describe the attraction forces among particles. We calculate the coordination number by

8 counting the number of particle if the surface distance of two particles is less than $2 \mathrm{~nm}$. We

9 verify that the coordination number is not considerably influenced if the surface

distance varies within a few $\mathbf{n m}$. Different from the fractal aggregate, the non-fractal

aggregate may have its internal connectivity vary. Therefore, the non-fractal aggregate used

in the study has different initial structures from loose to dense, equivalent to the initial average coordination number from 2.00 to 5.54 . The aggregates at these extreme conditions are given in Fig.1. The most dense aggregate has the coordination number $k_{0}=5.54$ somewhat smaller than that of random closed-packed particles around 5.9-8.5 [24-26]. This is because the number of particle of the aggregate is only one hundred, much smaller than the one that used in the literature from thousands to ten thousands particles. As a result, the particles near the aggregate surface, which have fewer surrounding particles than the inner ones do, bring about the decrease in the average coordination number.

\section{Results and discussion}

Figure 2 shows the instantaneous structures of various aggregates at the same shear condition $\mu \dot{\gamma}=26.9 \mathrm{~Pa}$. The loose aggregate with the initial coordination number $k_{0}=2.00$ deforms immediately while it keeps rotating around the vorticity axis. The deformation is complicated including stretching, compressing and bending; then the deformation gradually 
1 decreases after several rotations and the shape of the aggregate is eventually maintained. In

2 comparison, the more dense aggregate $k_{0}=3.02$ slightly deforms to an ellipsoid and quickly

3 recovers to a more spherical structure closed to the initial one. The most dense aggregate $k_{0}=$

45.54 retains its outer shape and rotates almost identically with the flow whose non-

5 dimensional time per rotation is $\dot{\gamma} t=4 \pi$. Preliminary observation reveals that although the

6 aggregates are non-fractal, the restructuring behaves differently depending on the

7 connectivity of the primary particles. The more dense aggregate has higher resistance to shear

8 flow and obtains stable structure faster than the loose aggregate does.

9 In order to investigate the details of the restructuring of aggregates, we examine the change in internal structure. Figure 3 presents the coordination number $k$ of aggregates with scaled time $\dot{\gamma} t$, at various shear flow conditions, for (a) dense aggregate, and (b) loose aggregate. The figure exhibits many similarities about the restructuring behavior, despite of different kind of profiles for the dense aggregate $\left(k_{0}=5.54\right)$ and the rather loose aggregate $\left(k_{0}\right.$ $=3.02$ ). The nature of the restructuring can be evidently explained by the data at $\mu \dot{\gamma}=26.9$ Pa. For this condition, the evolution of coordination number clearly shows that the aggregate restructures with two time scales. The short-term deformation, exhibited by the periodic fluctuation of coordination number, is explained by the passing of aggregate through the principal axes of stress tensor of simple shear flow twice per rotation. Via such short-term deformation, it is inferred that any non-fractal aggregate undergoes restructuring if shear flow is applied. In longer time scale, the coordination number levels off and approaches stable value $k_{s}$ as identified in the figures. The existence of stable coordination number coupling with the periodic fluctuation indicates that the stable state of aggregate is dynamic equilibrium in nature, resulting from the short-term deformation.

The change in coordination number with time and shear stress in Fig. 3 is analogical to the behavior of dense particle cluster studied by Harada et al. [13] as mentioned elsewhere. 
1 According to their results, the restructuring in terms of change in coordination number

2 includes three stages: (I) the cluster quickly gains a stable structure equivalent to the stable

3 coordination number, (II) the coordination number linearly decreases due to formation of

4 cracks, and (III) the coordination number rapidly decreases before the breakup of cluster. The

5 profiles of $k$ in Fig. 3 a for $\boldsymbol{k}_{\mathbf{0}}=\mathbf{5 . 5 4}$ are in good agreement. The coordination number shifts

6 from $k_{0}$ to $k_{s}$ in a short time within one rotation (stage I). The slopes in stage II depends on the

7 shear condition, when $\mu \dot{\gamma}=26.9 \mathrm{~Pa}$, stage II is very long and the profile almost maintains at

8 the value of $k_{s}$. For higher shear stress $\mu \dot{\gamma}=44.9 \mathrm{~Pa}$, the constant region of $k_{s}$ is followed by a

9 decreasing trend. As increasing $\mu \dot{\gamma}$ from $44.9 \mathrm{~Pa}$ to $59.8 \mathrm{~Pa}$, the decreasing trend is steeper than the previous one. Additionally, an irregular change corresponding to stage III is observed. In general, the stable coordination number in such cases are easily recognized because stage II is much longer than stage I, and the region of constant $k_{s}$ is explicitly given. The change in $k_{s}$ with $\mu \dot{\gamma}$ while the aggregate keeps it spherical shape demonstrates that the applied shear flow strongly affects the inner connectivity of the aggregate.

The results of loose aggregate $\left(\boldsymbol{k}_{\mathbf{0}}=\mathbf{3 . 0 2}\right)$ from Fig. $3 \mathrm{~b}$ show that at $\mu \dot{\gamma}=26.9 \mathrm{~Pa}$, the coordination number $k$ varies then approaches stable value, which is considered as stage I and stage II respectively. Different from dense aggregate, here the stage I prolongs while the deformation of the outer shape of this loose aggregate takes place. However, toward the end of stage I, little or no deformation is observed, and the shape of the aggregate at the end of stage I is almost spherical (see also Fig. 2). At higher shear stress $\mu \dot{\gamma}=44.9 \mathrm{~Pa}$, the profile expresses that stage II lasts for a short time compared to stage I, and there is a rapid change of coordination number to the breakup (stage III). For $\mu \dot{\gamma}=59.8 \mathrm{~Pa}$, stage II and stage III are not clearly distinguished. Moreover, we observe that in the two later cases of shear stress, the aggregate at the end of stage I has an irregular shape instead of a spherical one. The findings 
1 suggest that the restructuring of loose aggregate toward stable state is disrupted by the

2 breakup process when the shear stress is higher than a critical one, which is somewhere

3 between $26.9 \mathrm{~Pa}$ and $44.9 \mathrm{~Pa}$ in the condition of Fig. $3 \mathrm{~b}$ for example. Therefore, the stable

4 coordination number is also affected and difficult to determine in these cases. Since the aim

5 of our research is about the restructuring of aggregate, we mainly focus on the case that a

6 stable structure can be clearly obtained, i.e. stage II is longer than stage I and a spherical

7 shape at the end of stage I is accomplished. For such behavior, we consider that the

8 restructuring is dominant over breakup. The relation of stable coordination number with

9 initial coordination number and shear condition is discussed at the later part of the paper.

10 Figure 4 exhibits the evolution of coordination number of various aggregates under the

11 same condition of shear flow when the restructuring is dominant. Although the initial

12 structures of the aggregates are significantly different, their coordination numbers converge to

13 a specific narrow range whose upper limit of $k_{s}$ is determined by the groups of dense

14 aggregates having $k_{0}=5.54$ and $k_{0}=5.04$, and the lower limit by the aggregates having $k_{0} \leq$

15 4.20. The findings emphasize the important role of the restructuring in the construction of

16 breakup model which is strongly based on the aggregate structure.

17 A model is proposed to elucidate the restructuring of aggregate, i.e., the change in 18 coordination number with time. The net rate of change in coordination number is considered 19 as the sum of the formation rate and the disintegration rate of bonds connecting a particle to 20 its surrounding ones. We assume that all particles in the aggregate obey the same rule and 21 then the temporal change of the coordination number is described as follows:

$$
\frac{d k}{d(\dot{\gamma} t)}=\frac{d k^{+}}{d(\dot{\gamma} t)}+\frac{d k^{-}}{d(\dot{\gamma} t)}
$$

The rate of the formation and the disintegration of bonds are assumed to follow the linear 
$1 d k^{-} / d(\dot{\gamma} t)=-K^{-} k$ respectively. $K^{+}$and $K^{-}$are coefficients of proportionality and $k_{\max }$ is

2 the maximum coordination number which is set to be 6 , which is a generally accepted value

3 for random packed mono-spheres [25]. Integrating Eq.(5) from $\dot{\gamma} t=\mathbf{0}$ to arbitrary $\dot{\gamma} t$

4 gives us the analytical solution below.

$5 \quad k=\frac{K^{+} k_{\max }}{K^{+}+K^{-}}-\left(\frac{K^{+} k_{\max }}{K^{+}+K^{-}}-k_{0}\right) e^{-\left(K^{+}+K^{-}\right) \dot{\gamma t}}$

where $k_{0}$ is the initial coordination number of aggregate. Figure 5 shows the model results obtained from Eq.(6) by least-squares fitting method. The data employed in the fitting are limit from the initial value to the constant region. The model describes well the simulation results for all aggregate whose initial structure varies from loose to dense and even in the case that the restructuring is partially affected by breakup. Therefore, the restructuring of nonfractal aggregates can be explained by our proposed mechanism. Furthermore, the stable state of aggregate is considered dynamic equilibrium obtained when the formation rate and the disintegration rate are equal.

As we mention elsewhere about the importance of stable structure of aggregate, the relation between the stable coordination number $k_{s}$ and the external shear stress is given in Figure 6. The stable coordination number is constant at low shear stress while linearly decreases at high shear stress. The additional data in the gray area are the cases that the restructuring is affected by the breakup, as pointed out by the mix of stage II and stage III in Fig. $3 b$. Here we examine the behavior of $\boldsymbol{k}_{s}$ when the restructuring is dominant, i.e. the constant region and the linear decrease region.

In the low regime of $\mu \dot{\gamma}$ where the stable coordination number shows constant values, two identified groups are determined in Fig. 6. For the dense aggregates with $k_{0}=5.54$ and $k_{0}=5.04$, the stable coordination numbers are constant at 6.1 which is the highest value in all investigated conditions, whereas the aggregates with $k_{0} \leq 4.20$ obtain the slightly lower value 
1 at 5.92. In order to explain such a difference of stable coordination number in low shear stress,

2 we investigate the volume fraction of aggregate from a local viewpoint of each particle. The

3 particle volume fraction is defined as the ratio of the particle's volume to its Voronoi cell

4 [23]. Figure 7 presents the volume fraction of particles along the distance from the

5 aggregate's centroid, at initial state and stable state when shear stress is low. The trend of the

6 data along the radius of aggregate is similar in all cases, which is illustrated by the relatively

7 uniform volume fraction at the inner part, and the dramatic decrease values at the outer part

8 that more exposes to the surrounding fluid. Here we only focus on the data from the inner part

9 of the aggregate. The dense aggregates with $k_{0}=5.54$ and $k_{0}=5.04$ exhibit that the volume

10 fraction is almost identical between the stable state and initial state whose volume fraction is

11 around the value of random close packed structure. On the other hand, the aggregates in

the case $k_{0} \leq 4.20$ show that, the stable state is more compact than the initial one, but not as compact as the aforementioned dense aggregate. These findings mean that, for the dense aggregates, the particles are trapped among their neighbors, and move restrictively within the existed void during restructuring. Therefore, the structure of the dense aggregate is considered static equilibrium. In opposite, the initial volume fraction of the loose aggregates with $k_{0} \leq 4.20$ reflects unstable structures. Under the external shear stress, the particles restructure, form more connections, and the aggregate eventually becomes more dense with time. As the volume fraction increases, the void among the particle also reduces, leading to the inability of particles to explore the space around them. The state that the particles are no more capable of occupying their surrounding space is regarded as quasistable whose volume fraction is lower than that of random close packing [27]. In general, the results of Fig. 7 reveal that the dependence of stable structure on initial coordination number originated from the irreversible transition from quasi-stable structure to static-equilibrium, which also explains the small gap of $k_{s}$ shown in Fig. 6 when shear stress is low. 
Regarding the linear decreasing trend of $\boldsymbol{k}_{\boldsymbol{s}}$ with $\mu \dot{\gamma}$ in Fig. 6, such a tendency arises

2 from the dependence of the mobility of particles on the surrounding flow. The higher shear

3 stress is, the more mobile particles are, while the attractive force is independent of flow

4 condition. Consequently, it is easier for a bond between particles to be cut. As the

5 aggregate approaches dynamic equilibrium, i.e. the formation and the disintegration of

6 bonds becomes equal, the $k_{s}$ is not as high as the $k_{s}$ obtained from low shear stress. In

7 addition, the similar slopes of all cases reveal that the effect of shear stress on the change in

8 connectivity of stable state is the same, not depending on the initial structure of aggregate.

9

10

11

12

\section{Conclusion}

Restructuring behavior of non-fractal aggregates with different connectivity in shear flow has been performed numerically. The time evolution of internal structure of aggregate for various flow conditions is investigated. Under the rotation and deformation effect of shear flow, non-fractal aggregates undergo restructuring and attain the stable structure, which can be explained by the dynamic formation and disintegration of bonds inside the aggregate. The stable structure of aggregate is strongly dependent on applied shear but weakly dependent on initial structure.

\section{Acknowledgement}

We are grateful to National Institute of Standards and Technology for providing the computer program of Voronoi tessellation.

\section{References}

[1] Y.M. Harshe, M. Lattuada, M. Soos, Experimental and modeling study of breakage and restructuring of open and dense colloidal aggregates, Langmuir, 27 (2011) 5739-5752. 
1 [2] S.P. Rwei, I. Manas-Zloczower, D.L. Feke, Observation of carbon black agglomerate 2 dispersion in simple shear flow, Polym. Eng. Sci., 30 (1990) 701-706.

3 [3] S. Blaser, Flocs in shear and strain flows, J. Colloid Interface Sci., 225 (2000) 273-284.

4 [4] S. Harada, R. Tanaka, H. Nogami, M. Sawada, Dependence of fragmentation behavior of 5 colloidal aggregates on their fractal structure, J. Colloid Interface Sci., 301 (2006) 123-129.

6 [5] V. Becker, E. Schlauch, M. Behr, H. Briesen, Restructuring of colloidal aggregates in 7 shear flows and limitations of the free-draining approximation, J. Colloid Interface Sci., 339 8 (2009) 362-372.

9 [6] M.L. Eggersdorfer, D. Kadau, H.J. Herrmann, S.E. Pratsinis, Fragmentation and restructuring of soft-agglomerates under shear, J. Colloid Interface Sci., 342 (2010) 261-268. [7] B.O. Conchuir, Y.M. Harshe, M. Lattuada, A. Zaccone, Analytical model of fractal aggregate stability and restructuring in shear flows, Ind. Eng. Chem. Res., 53 (2014) 91099119.

[8] S. Horwatt, I. Manas-Zloczower, D.L. Feke, Dispersion behavior of heterogeneous agglomerates at supercritical stresses, Chem. Eng. Sci., 47 (1992) 1849-1855.

[9] K. Higashitani, K. Iimura, H. Sanda, Simulation of deformation and breakup of large aggregates in flows of viscous fluids, Chem. Eng. Sci., 56 (2001) 2927-2938.

[10] C. Selomulya, G. Bushell, R. Amal, T.D. Waite, Understanding the role of restructuring in flocculation: The application of a population balance model, Chem. Eng. Sci., 58 (2003) 327-338.

21 [11] L. Gmachowski, Aggregate restructuring and its effect on the aggregate size distribution, 22 Colloids and Surfaces A: Physicochem. Eng. Aspects, 207 (2002) 271-277. 
1 [13] S. Harada, R. Tanaka, H. Nogami, M. Sawada, K. Asakura, Structural change in non-

2 fractal particle clusters under fluid stress, Colloids and Surfaces A: Physicochem. Eng. 3 Aspects, 302 (2007) 396-402.

4 [14] J.F. Brady, G. Bossis, Stokesian dynamics, Ann. Rev. Fluid Mech., 20 (1988) 111-157.

5 [15] L. Durlofsky, J.F. Brady, G. Bossis, Dynamic simulation of hydrodynamically

$6 \quad$ interacting particles, J. Fluid Mech., 180 (1987) 21-49.

7 [16] D.J. Jeffery, Y. Onishi, Calculation of the resistance and mobility functions for two 8 unequal rigid spheres in low-Reynolds-number flow, J. Fluid Mech., 139 (1984) 261-290.

9 [17] S. Kim, S.J. Karrila, Microhydrodynamics, Butterworth-Heinemann, Boston, 1991.

10 [18] H.C. Hamaker, The London-van der Waals attraction between spherical particles, 11 Physica, 4 (1937) 1058-1072.

[19] W.R. Bowen, F. Jenner, The calculation of dispersion forces for engineering application, Adv. Colloid Interface Sci., 56 (1995) 201-243.

[20] J.N. Israelachvili, Intermolecular and Surface Force, Academic Press, London, 1992.

[21] Y.M. Harshe, M. Lattuada, Breakage rate and colloidal aggregates in shear flow through Stokesian dynamics, Langmuir, 28 (2012) 283-292.

[22] L.F. Liu, Z.P. Zhang, A.B. Yu, Dynamic simulation of the centripetal packing of monosized spheres, Physica A, 268 (1999) 433-453.

[23] B. Cambou, M. Jean, F. Radjaï, Micromechanics of Granular Materials, John Wiley \& Son, 2013.

[24] J.D. Bernal, J. Mason, Packing of spheres: Co-ordination of randomly packed spheres, Nature, 188 (1960) 910-911.

[25] G.T. Nolan, P.E. Kavanagh, Computer simulation of random packing of hard spheres, Powder Technol., 72 (1992) 149-155. 
1 [26] S.R. Williams, A.P. Philipse, Random packings of spheres and spherocylinders

2 simulated by mechanical contraction, Phys. Rev. E, 67 (2003) 051301-1-9.

3 [27] T. Aste, Variations around disordered close packing, J. Phys.: Condens. Matter, 17 4 (2005) S2361-S2390. 


\section{Figures}

Fig. 1 The coordinate system and initial configurations of aggregates $k_{0}=2.00$ (left) and $k_{0}=5.54$ (right)

Fig. 2 Instantaneous motion of aggregates with nondimensional time at $\mu \dot{\gamma}=26.9 \mathrm{~Pa}$ for (a)

$$
k_{0}=2.00, \text { (b) } k_{0}=3.02, \text { (c) } k_{0}=5.54 \text {. }
$$

Fig. 3 Coordination number of aggregate at different shear stress conditions for (a) $k_{0}=5.54$,

(b) $k_{0}=3.02$.

Fig. 4 Coordination number of aggregates during restructuring at shear stress $\mu \dot{\gamma}=26.9 \mathrm{~Pa}$.

Fig. 5 Modeling the coordination number of aggregates at different shear stress conditions.

Fig. 6 Relation between shear stress and stable coordination number of aggregates.

Fig. 7 Radial distribution of particle volume fraction at initial structure and stable structure of aggregates at condition $\mu \dot{\gamma}=12.0 \mathrm{~Pa}$. 


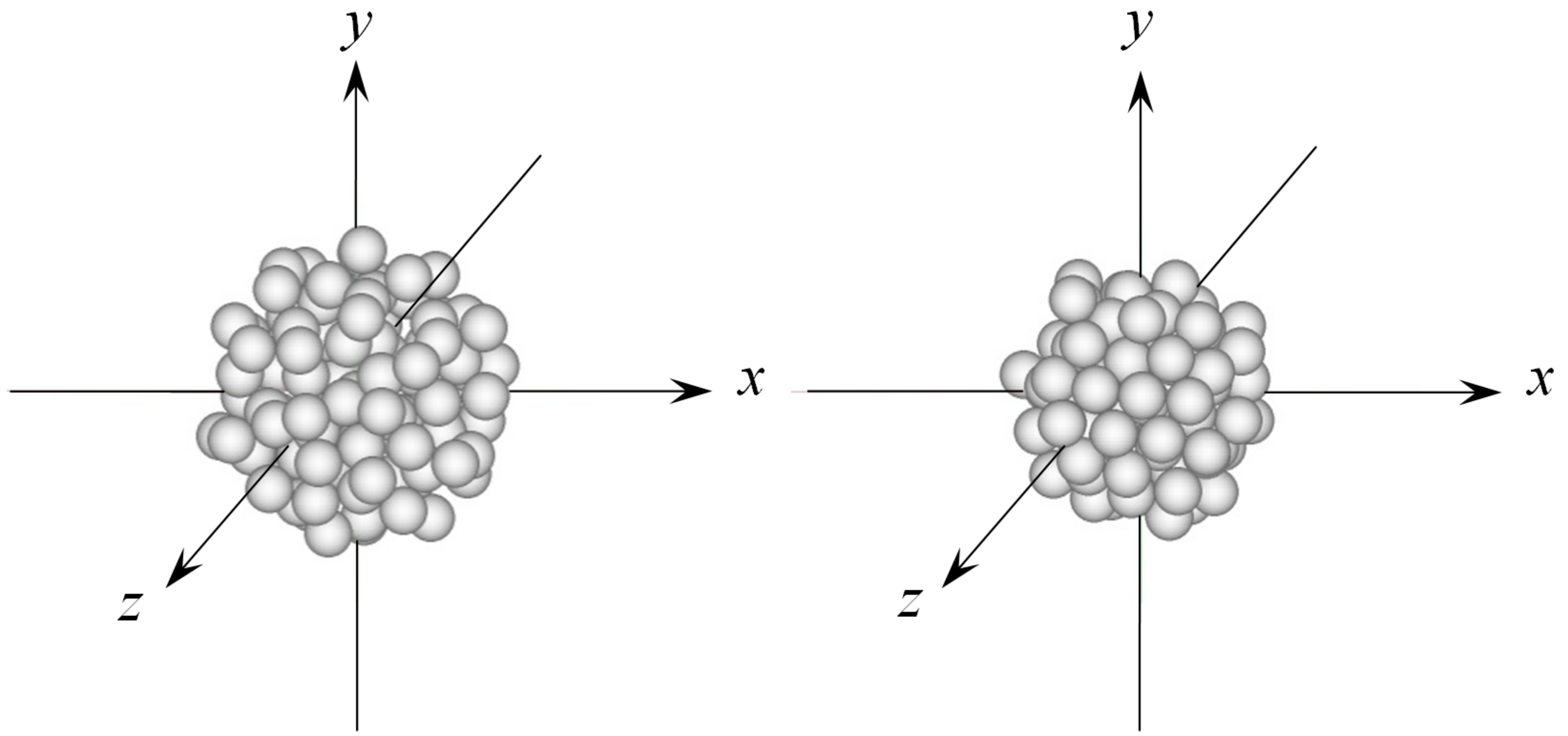


Figure 2

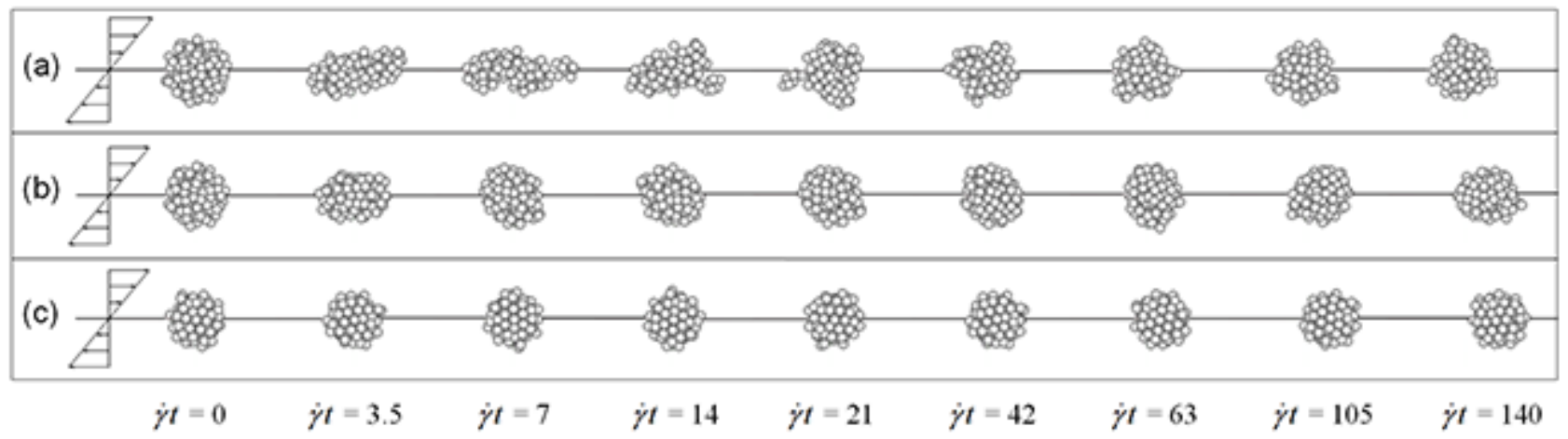



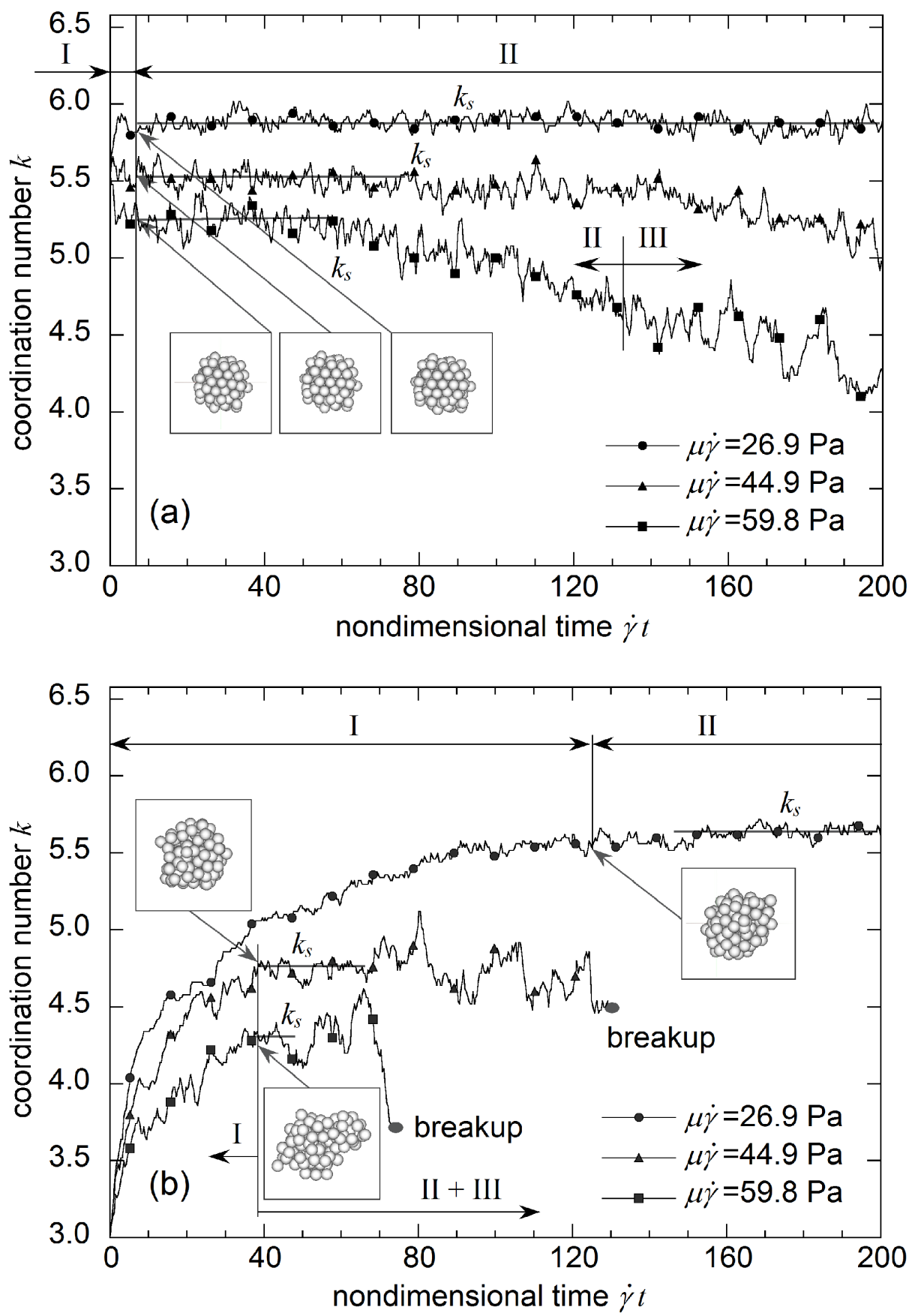


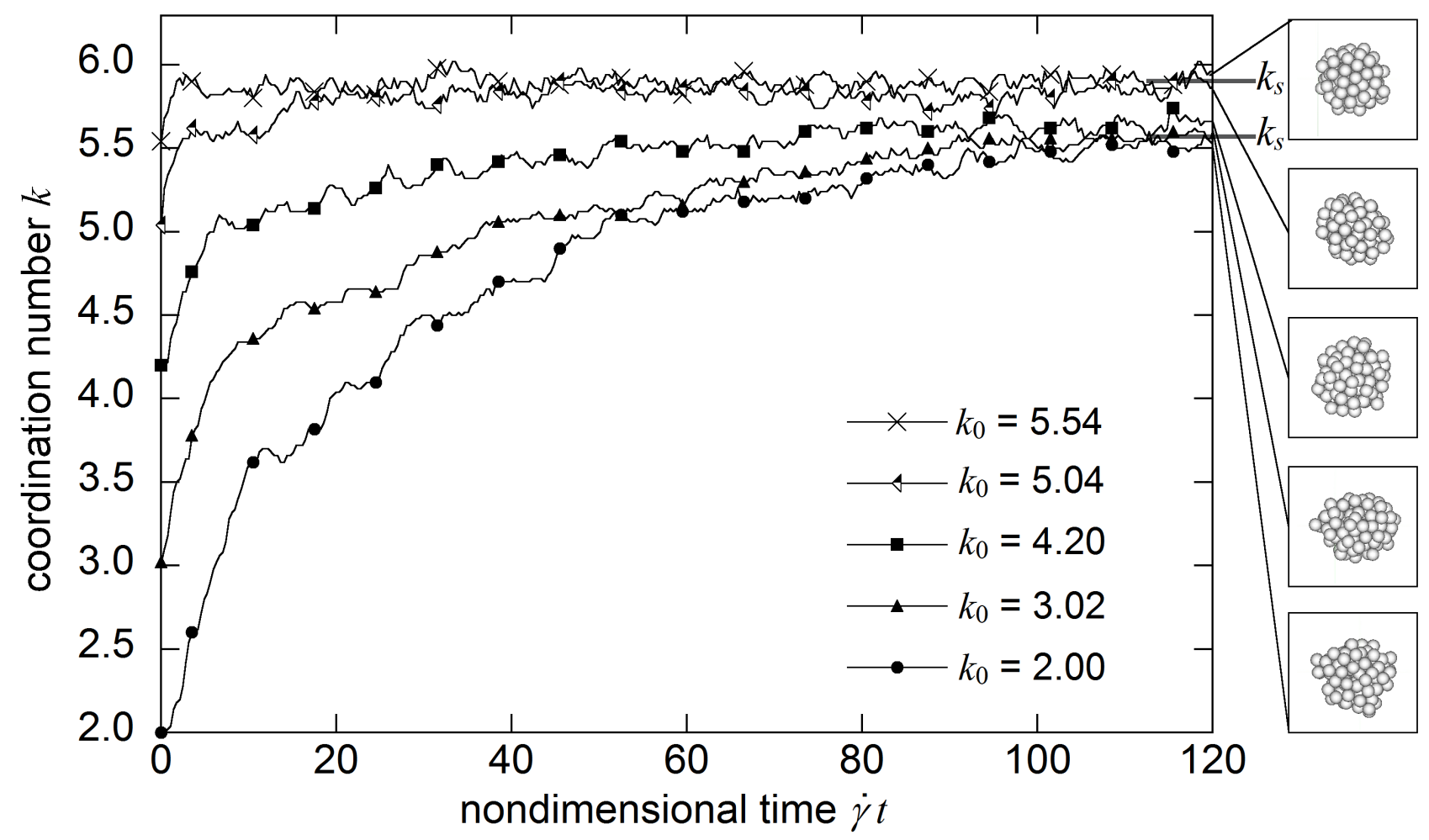




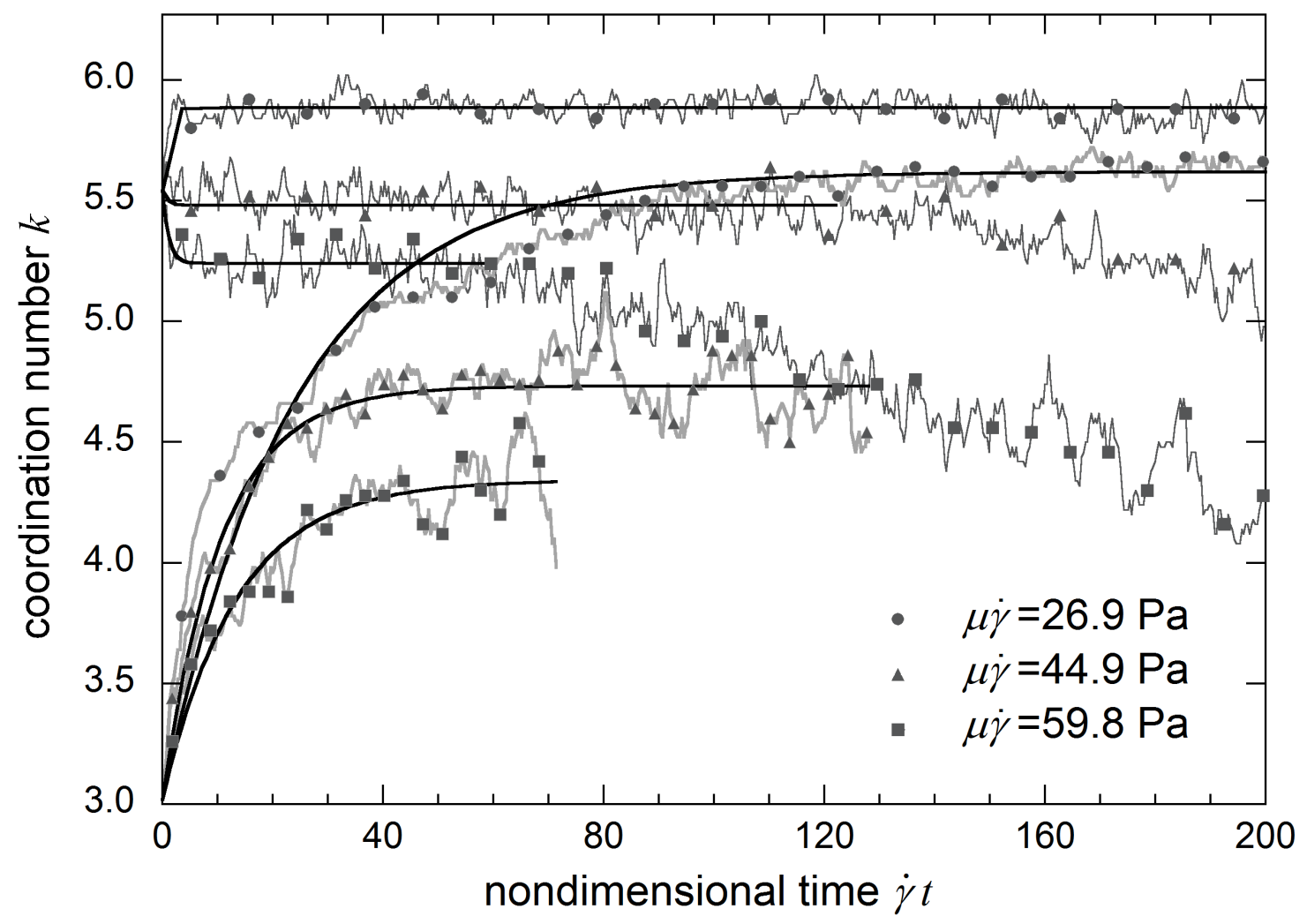




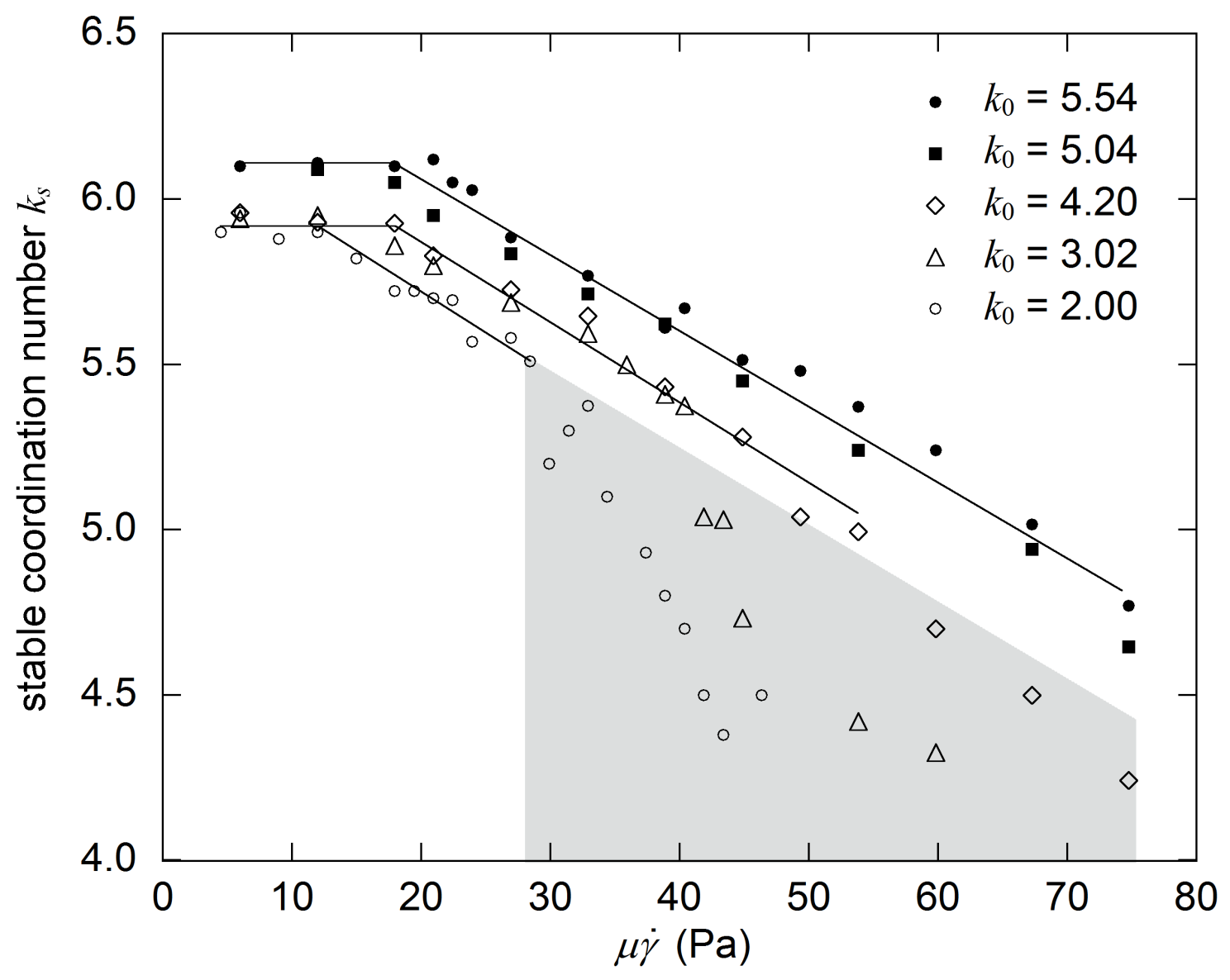



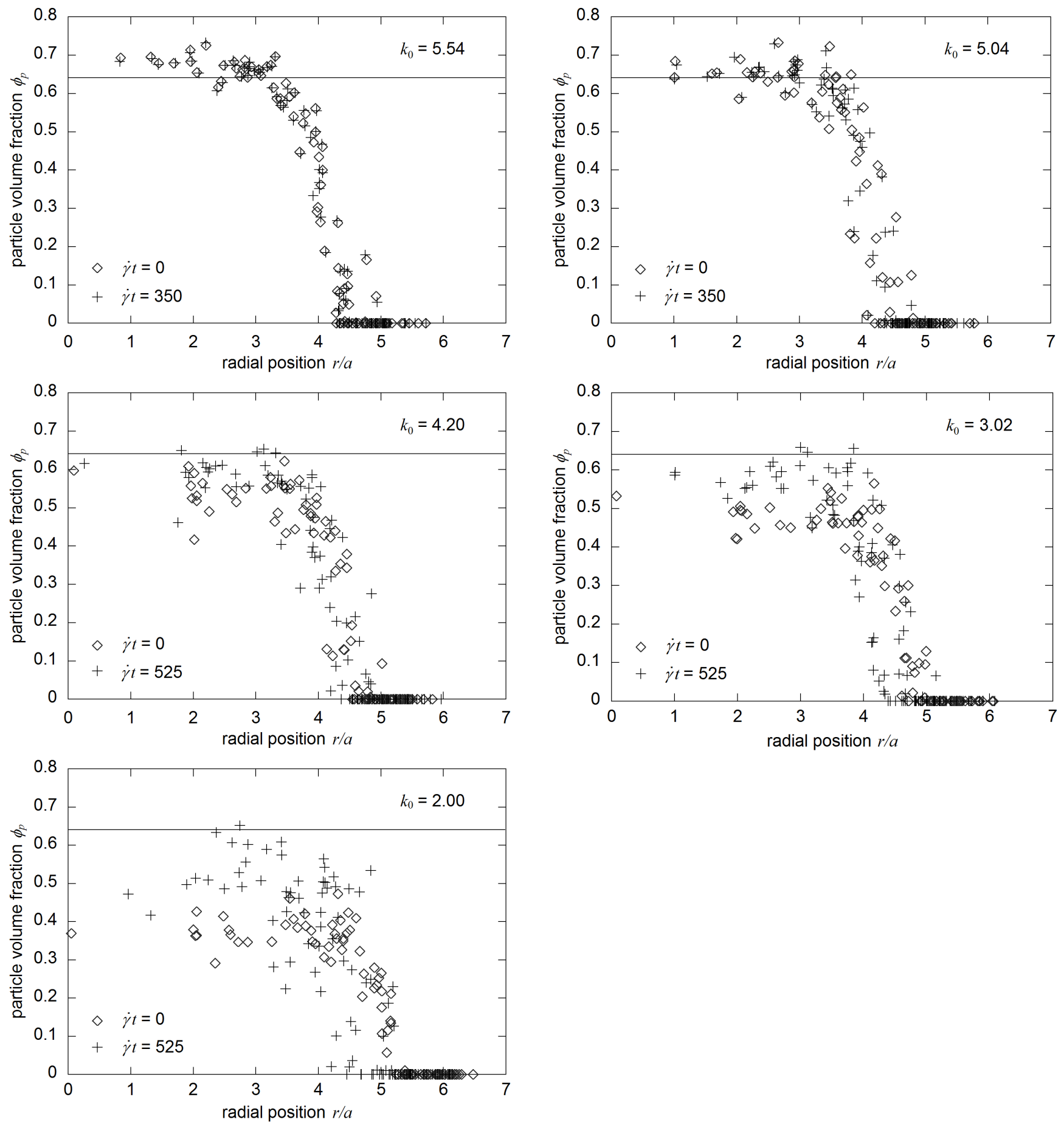